\title{
Assessing Bos taurus introgression in the UOA Bos indicus assembly
}

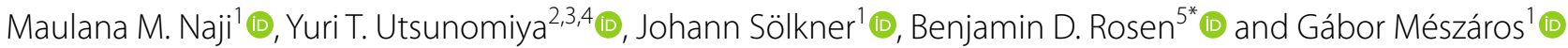

\begin{abstract}
Background: Reference genomes are essential in the analysis of genomic data. As the cost of sequencing decreases, multiple reference genomes are being produced within species to alleviate problems such as low mapping accuracy and reference allele bias in variant calling that can be associated with the alignment of divergent samples to a single reference individual. The latest reference sequence adopted by the scientific community for the analysis of cattle data is ARS_UCD1.2, built from the DNA of a Hereford cow (Bos taurus taurus-B. taurus). A complementary genome assembly, UOA_Brahman_1, was recently built to represent the other cattle subspecies (Bos taurus indicus_-B. indicus) from a Brahman cow haplotype to further support analysis of $B$. indicus data. In this study, we aligned the sequence data of 15 B. taurus and $B$. indicus breeds to each of these references.

Results: The alignment of $B$. taurus individuals against UOA_Brahman_1 detected up to five million more singlenucleotide variants (SNVS) compared to that against ARS_UCD1.2. Similarly, the alignment of B. indicus individuals against ARS_UCD1.2 resulted in one and a half million more SNVs than that against UOA_Brahman_1. The number of SNVs with nearly fixed alternative alleles also increased in the alignments with cross-subspecies. Interestingly, the alignment of B. taurus cattle against UOA_Brahman_1 revealed regions with a smaller than expected number of counts of SNVs with nearly fixed alternative alleles. Since B. taurus introgression represents on average $10 \%$ of the genome of Brahman cattle, we suggest that these regions comprise taurine DNA as opposed to indicine DNA in the UOA_Brahman_1 reference genome. Principal component and admixture analyses using genotypes inferred from this region support these taurine-introgressed loci. Overall, the flagged taurine segments represent $13.7 \%$ of the UOA_Brahman_1 assembly. The genes located within these segments were previously reported to be under positive selection in Brahman cattle, and include functional candidate genes implicated in feed efficiency, development and immunity.
\end{abstract}

Conclusions: We report a list of taurine segments that are in the UOA_Brahman_1 assembly, which will be useful for the interpretation of interesting genomic features (e.g., signatures of selection, runs of homozygosity, increased mutation rate, etc.) that could appear in future re-sequencing analysis of indicine cattle.

\section{Background}

Reference genome assemblies are linear haploid sequences that serve as a standard for the analysis of all genomic data and databases $[1,2]$. They provide a

*Correspondence: ben.rosen@usda.gov

${ }^{5}$ Animal Genomics and Improvement Laboratory, USDA, ARS, Beltsville, MD, USA

Full list of author information is available at the end of the article framework for variant calling, RNA and other sequencing read alignments, gene annotation, and functional analyses [1]. In common practice, sequencing reads undergo a pipeline that starts with the alignment against the reference genome followed by various downstream analyses depending on the respective aims of the study. In a later stage, the loci of interest obtained from the analyses will be annotated back to the same reference genome to infer the genes that are found at these loci and their original author(s) and the source, provide a link to the Creative Commons licence, and indicate if changes were made. The images or other third party material in this article are included in the article's Creative Commons licence, unless indicated otherwise in a credit line to the material. If material is not included in the article's Creative Commons licence and your intended use is not permitted by statutory regulation or exceeds the permitted use, you will need to obtain permission directly from the copyright holder. To view a copy of this licence, visit http://creativecommons.org/licenses/by/4.0/. The Creative Commons Public Domain Dedication waiver (http://creativeco mmons.org/publicdomain/zero/1.0/) applies to the data made available in this article, unless otherwise stated in a credit line to the data. 
functionalities [3-5]. A high level of genetic variation within a species can lead to a lower mapping rate of reads and reference allele bias in variant calling if only a single reference genome is used. Thus, as DNA sequencing costs decrease and novel methodologies emerge, multiple reference genomes are being generated for the same species to attempt to rectify previous drawbacks of the use of single reference genomes [6-8].

The total number of gaps in a genome is one indicator for assessing the quality of de-novo reference assemblies. For example, the current reference human genome version 38 (also known as GRCh38) features around 650 gaps $[1,7]$. The latest and most widely used cattle genome release ARS_UCD1.2 has only 386 gaps in its final assembly. This assembly was an update of the UMD3.1 assembly that was based on the same inbred Hereford cow as the DNA source providing $250 \times$ more continuity than its predecessor $[9,10]$. In addition, there are two assemblies built from a single F1 cross of a Brahman (Bos taurus indicus-referred as $B$. indicus hereafter) dam with an Angus (Bos taurus taurus-referred as B. taurus onwards) bull. Using parent-specific k-mers, the paternal and maternal haplotypes of the F1 animal were separated, leading to an Angus-specific assembly (UOA_Angus_1) and a Brahman-specific assembly (UOA_Brahman_1). Both assemblies were constructed by combining the latest sequencing technologies of PacBio long-reads, Hi-C data, Bio-nano optical reads and Illumina short reads, respectively resulting in 277 and 302 gaps in the final assemblies, which is less than in GRCh38 and ARS UCD1.2 $[7,9]$. It should be noted that the UOA_Brahman_1 reference sequence is the first published de novo assembly of a $B$. indicus genome.

Although Brahman is considered to be a $B$. indicus breed, its formation has a history of admixture between several $B$. indicus and $B$. taurus breeds $[11,12]$. It has been estimated that the current Brahman population retains $10 \%$ of $B$. taurus ancestry on average [12]. Part of this taurine ancestry has been retained due to artificial selection for the introgression of productivity and reproduction-related traits from those $B$. taurus breeds into the indicine background [11]. Conversely, the high indicine content in the Brahman genome ensures that this breed exhibits most of the traits that are considered beneficial in hot and humid climates, such as heat tolerance and resistance to parasites and infectious diseases $[12,13]$.

The main objective of using a $B$. indicus instead of a $B$. taurus assembly to guide alignments of re-sequencing data of indicine cattle is to mitigate bias in variant calling. Since $B$. taurus and $B$. indicus diverged about 300,000 years ago, mutation and drift fostered the accumulation of many base substitutions and structural variations between the two subspecies, which in practice may lower the alignment performance of short sequence reads $[14,15]$. Indeed, the percentage of indicine reads that are properly aligned against a taurine reference genome has been found to be slightly lower as compared to taurine re-sequencing data $[16,17]$. In addition, the number of variants detected in indicine data tends to surpass that of taurine data by millions of variants, which indicates assembly bias during variant calling [18]. In this study, our aims were to (1) examine possible reference genome bias by comparing the number of variant sites detected when using the ARS_UCD1.2 and UOA_Brahman_1 reference sequences; (2) identify genomic regions containing traces of $B$. taurus introgression into the UOA_Brahman_1 assembly; and (3) explore the genes within these regions of interest that may have been selectively retained.

\section{Methods \\ Datasets}

We used datasets from NCBI retrieved in FASTQ format from the mirror server at ebi.ac.uk. The dataset consists of $B$. taurus and $B$. indicus cattle from several breeds as listed in Table 1; details for each individual are provided in Additional file 1: Table S1. In total, we used wholegenome sequence data on 112 individuals $(54 \mathrm{~B}$. taurus and $58 \mathrm{~B}$. indicus) from 15 breeds (6 B. taurus and $9 B$. indicus).

\section{Alignments and single nucleotide variant (SNV) calling}

We used the Burrows-Wheeler Aligner (BWA) v.0.7.17 [19], with the maximal exact matches (mem) algorithm and default parameters for mapping paired-end reads to a reference genome. Then, aligned reads in sequence alignment map (SAM) files were sorted by chromosome and consecutively converted to binary alignment map (BAM) files using the sort function of samtools v1.10 [20]. Duplicate reads in each BAM file were flagged using the Picard (https://broadinstitute.github.io/picard/) MarkDuplicates tool embedded in the genome analysis tool kit (GATK v.4.1.0) [21]. Read groups were modified accordingly using Picard AddOrReplaceReadGroups. We applied the BaseRecalibrator and ApplyBQSR functions of GATK v.4.1.0 for base quality score recalibration of each BAM file. We called the genomic variant call format (GVCF) file using the GATK v.4.1.0 HaplotypeCaller with the -ERC GVCF option for each BAM file. We combined the GVCF of samples for each breed using GATK v.4.1.0 CombineGVCFs and subsequently performed GenotypeGVCF with an output of variant call format (VCF) for each respective breed. We performed SplitVcfs to extract SNVs and VariantFiltration to filter variants with the following parameters 
Table 1 List of FASTQ reads dataset

\begin{tabular}{|c|c|c|c|c|c|c|c|c|c|c|}
\hline \multirow[t]{2}{*}{ Breeds } & \multirow[t]{2}{*}{$\mathbf{N}$} & \multirow[t]{2}{*}{ Read length } & \multirow[t]{2}{*}{ Total reads } & \multirow[t]{2}{*}{ Cov } & \multicolumn{3}{|c|}{ ARS_UCD1.2 (\%) } & \multicolumn{3}{|c|}{ UOA_Brahman_1 (\%) } \\
\hline & & & & & Map & Filt & Ret & Map & Filt & Ret \\
\hline Angus ${ }^{a}$ & 9 & 151 & 172 & 7.26 & 97.65 & 13.74 & 83.91 & 97.77 & 14.99 & 82.77 \\
\hline Hereford $^{\mathrm{a}}$ & 8 & 99 & 242 & 10.17 & 99.34 & 15.83 & 83.51 & 99.47 & 17.26 & 82.21 \\
\hline Holstein ${ }^{a}$ & 9 & 100 & 124 & 4.58 & 93.37 & 16.82 & 76.55 & 93.45 & 17.91 & 75.54 \\
\hline Jersey $^{a}$ & 7 & 100 & 186 & 6.86 & 98.79 & 19.79 & 79.00 & 98.84 & 20.84 & 78.00 \\
\hline Shorthorn ${ }^{a}$ & 5 & 99 & 230 & 8.36 & 99.06 & 19.06 & 80.00 & 99.13 & 20.33 & 78.80 \\
\hline Simmental ${ }^{a}$ & 16 & 100 & 217 & 8.59 & 92.91 & 17.02 & 75.89 & 92.98 & 18.28 & 74.70 \\
\hline Bohaibc $^{b c}$ & 3 & 150 & 222 & 12.21 & 99.55 & 25.12 & 74.44 & 99.68 & 26.40 & 73.28 \\
\hline Boran $^{b}$ & 10 & 101 & 297 & 11.03 & 99.54 & 15.49 & 84.04 & 99.52 & 15.94 & 83.58 \\
\hline Brahman $^{b}$ & 7 & 114 & 283 & 11.64 & 88.74 & 3.98 & 84.76 & 89.84 & 4.58 & 85.26 \\
\hline $\mathrm{Gir}^{\mathrm{b}}$ & 9 & 99 & 91 & 3.27 & 97.09 & 12.40 & 84.69 & 95.64 & 12.32 & 83.33 \\
\hline Indian zebu ${ }^{b}$ & 5 & 100 & 152 & 5.56 & 98.83 & 33.05 & 65.79 & 98.77 & 33.12 & 65.64 \\
\hline Kenanab & 6 & 101 & 307 & 11.40 & 99.50 & 14.59 & 84.91 & 99.51 & 14.68 & 84.83 \\
\hline Mangshib & 7 & 100 & 101 & 3.68 & 98.29 & 17.50 & 80.80 & 98.25 & 17.64 & 80.62 \\
\hline Nelore $^{b}$ & 6 & 99 & 111 & 4.00 & 98.18 & 13.35 & 84.83 & 98.05 & 13.85 & 84.20 \\
\hline Ogaden $^{b}$ & 5 & 101 & 291 & 10.80 & 99.51 & 15.65 & 83.86 & 99.49 & 16.11 & 83.38 \\
\hline
\end{tabular}

$\mathrm{N}$ : Number of individuals; Read length: read length values were derived from the mode of read length from individuals raw reads from each respective breed; Total reads: total reads in million; Cov: coverage values were estimated from total bases in raw reads and using $2.7 \mathrm{~Gb}$ as the genome length; Map: mapped values were percentage of reads aligned to the respective reference genome; Filt: filter values were percentage of reads dropped from the BAM files due to not passing the parameters set during the base recalibration step in the genome analysis tool kit (GATK); Ret: retain values were percentage of reads passing the GATK base recalibration step and kept in the final BAM files for the next step of variants calling for the respective reference genome; All values for total reads, coverage values, mapped values, filter values, and retain values are averages of individuals for each respective breed

${ }^{\text {a }}$ Bos taurus taurus

${ }^{\mathrm{b}}$ Bos taurus indicus

' Highly admixed with B. taurus ancestry

"QD < 2.0", "QUAL < 30.0", "MQ< 40.0", "SOR > 3.0", "FS > 60.0", "MQRankSum<-12.5", "ReadPosRankSum $<-8.0$ ". The general pipeline for handling wholegenome sequence (WGS) reads from raw FASTQ up to the variant filtration step, which resulted in a final VCF file for each breed, is shown in Fig. 1a. Unless specifically mentioned, default parameters were used for the tools applied in this study.

A VCF file template is required during the GATK v.4.1.0 BaseRecalibrator step to follow Best Practices as recommended by GATK [5]. This step detected and corrected possible systemic errors in base quality scores of mapped reads, particularly for bases near known variants. For the ARS_UCD1.2 pipeline, we used a VCF file provided by the 1000 bulls genome project [22]. Due to the unavailability of a VCF file template for UOA_Brahman_1 at the time of analysis, we generated an in-house raw VCF file called by bcftools mpileup [20] based on a random selection of five Indian zebu and Brahman individuals retrieved from previous studies [11, 23, 24] with accession numbers SRR6423815, SRR4279980, SRR4280002, SRR4280008, and SRR6650038. This template VCF file consisted of $\sim 17.5$ million variant sites across the genome.

\section{Hypothesis for the alignment to divergent reference genomes}

As indicated by previous studies [7, 18], alignment of individuals against a reference genome built using the same breed results in the detection of fewer SNVs compared to mapping those individuals against a reference genome built from another breed. For instance, consider individual $\mathrm{x}$ that belongs to breed $\mathrm{A}$ and individual $\mathrm{y}$ that belongs to breed $\mathrm{B}$, alignment of individual $\mathrm{x}$ against the $B$ reference genome will lead to more detected variants compared to that against the A reference genome and vice versa for individual $y$. Based on this assumption, we analysed the number of detected SNVs in two scenarios of alignment using ARS_UCD1.2 and UOA_Brahman_1.

\section{Analysis of SNV frequencies}

Pools of FASTQ files from the same breed were processed through the SNV calling pipeline, as shown in Fig. 1b. SNV frequencies were retrieved using vcftools [25] with the -freq 2 command, then we applied an 'awk' 


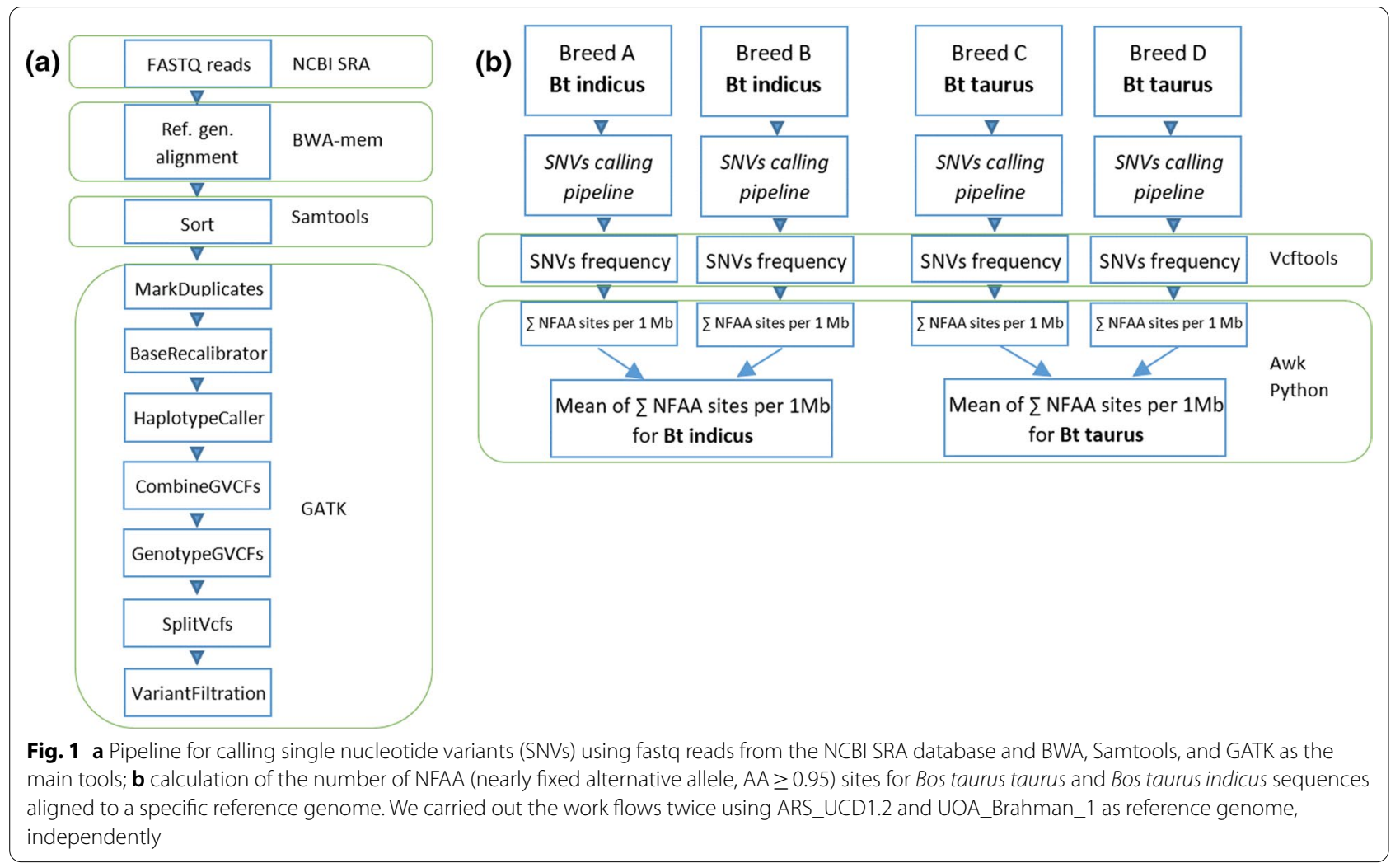

command to retain only the autosomal-diploid sites with a $100 \%$ call rate. Then, using an in-house script (nearly fixed alternative allele (NFAA) NFAA_comparisons. py), we retained only the SNVs with a frequency of the alternative allele equal to or higher than 0.95, i.e. SNVs that are almost homozygous for the alternative allele. All codes applied during the analysis are provided in https:// github.com/mas-agis/taurus_segment_uoa_brahman.git. For each breed, there were two frequency files, one using the ARS_UCD1.2 and one using the UOA_Brahman_1 assembly as the reference genome. The frequency file is a summary of SNV frequencies with chromosome name, position, reference allele, alternative allele, and the frequencies of reference and alternative alleles within each breed which were obtained as described previously.

As in the Notation 1 shown below, using non-overlapping $1-\mathrm{Mb}$ scanning windows, we sum the number of NFAA sites in both pipelines using the ARS_UCD1.2 and UOA_Brahman_1 assemblies. We plotted $A_{i}$ as the sum of NFAA sites across autosomal positions for both reference genome assemblies. Only in the case of the alignment of taurine cattle against the UOA_Brahman_1 assembly, was Notation 2 for window $i$ followed to calculate the delta value $\Delta_{i}$ (difference between average number and observed number NFAA sites in each scanning window) by subtracting the mean number of NFAA sites $\mu(A)$ from the total number of NFAA sites $A_{i}$ on the respective window $i$. In a single normal distribution, 95\% of the data are under $\mu \pm 1.96$ standard deviation (sd), however, the density function of all $\Delta$ values, Additional file 2: Fig. S1, depicts two peaks in the curve instead of a single normal distribution. Therefore, since we were most interested in the second (right) peak which has the largest deviation from the average, we used a threshold of $\mu$ $+1.5 \mathrm{sd}$. In Notation 3, $T$ denotes the putative taurineintrogressed regions and corresponds to the set of $\Delta$ values that are above our threshold of $\mu+1.5$ sd of all $\Delta$ values.

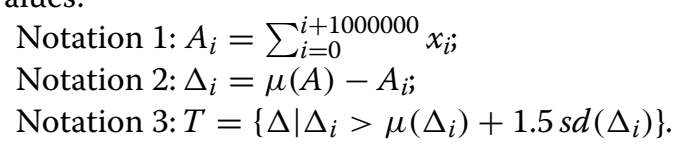

\section{Validation}

To validate the taurine-introgressed segments in the UOA_Brahman_1 assembly, we carried out principal components (PCA) and admixture analysis using SNVs as inputs. VCF files from the alignment of each breed against UOA_Brahman_1 were converted to plink format. Files from each breed were combined using the merge function of the Plink1.9 software [26]. We set the UOA_Brahman_1 reference genome as an individual that is homozygous for the reference allele at all variant 
positions (labelled as the Brahman haplotype hereafter) and merged it with the combined dataset. Since the Plink software handles only biallelic sites, we removed 9718 triallelic sites present in the dataset. We inferred the PCA using the plink function of -pca with preset 5 Eigenvalues from $11,135,811 \mathrm{SNV}$ s in the whole genome, $801,366 \mathrm{SNVs}$ in putative taurine introgressed regions and 10,334,445 SNVs in non-introgressed regions of the UOA_Brahman_1 assembly. Eigenvalues were plotted using ggplot2 [27] in R [28].

We used the Admixture v.1.3 software [29] to estimate the population structure of all the individuals and the Brahman haplotype that was inferred from SNVs derived from the whole genome, putative taurine-introgressed, and non-introgressed regions of the alignment against UOA_Brahman_1 as well as SNVs from the alignment of individuals against the ARS_UCD1.2 reference genome. In the Admixture software, we set the number of $\mathrm{K}$ to 3, which allowed a clear distinction between $B$. taurus and B. indicus. Admixture outputs were plotted using R [28].

\section{Functional gene annotation}

We examined the taurine-introgressed regions in the UOA_Brahman_1 assembly flagged in the previous steps to identify the genes they harbour. Physical genome locations of these segments were re-written to bed format, which includes chromosome number, start and end of the scanning window. The snpEFF software [30] annotated genes from the bed file using the annotation file of the UOA_Brahman_1 version 99 from Ensembl. Genes were assigned to a respective window if their transcripts were located within the window. The genes found within these taurine-introgressed regions were compared to a list of genes that were previously reported to be linked to B. taurus genome segments in influential Brahman bulls [11].

\section{Results}

Whole-genome sequence data on 112 individuals from six breeds of $B$. taurus and nine breeds of $B$. indicus ancestry were aligned against the ARS_UCD1.2 and UOA_Brahman_1 reference genomes. In this study, we considered the 29 autosomes with a total length of $2489 \mathrm{Mb}$ for ARS_UCD1.2 and $2478 \mathrm{Mb}$ for UOA_Brahman_1. Table 1 shows the percentages of total aligned reads to each reference genome (Map), percentages of reads removed during the base recalibration process from BAM files due to low mapping rate and duplicate reads in the alignment (Filt). Ret is the total percentage of reads kept in the final BAM files used for SNV calling for the two alignments against the ARS_UCD1.2 and UOA_ Brahman_1 reference genomes; details on the individuals included in the study are in Additional file 1: Table S1. A pairwise t-test showed that there was no significant difference $(p$-value $=0.53)$ in the total number of aligned reads (Map) between the two alignments of individuals to the reference genomes. However, the test was significant ( $p$-value $=0.0002)$ when we used the percentage of retained reads (Ret) since the number of reads retained in the final BAM files for all breeds, except Brahman, was smaller for the alignment against UOA_Brahman_1 than that against ARS_UCD1.2.

We called the SNVs for each breed, separately, to detect SNVs that might be unique to one breed. Table 2 shows the number of SNVs in each reference genome using the same quality parameters as for variant filtration. We performed pairwise t-tests for the total number of SNVs from Table 2, separately, for taurine and indicine cattle. A significant difference $(\mathrm{p}$-value $=0.0033$ ) in the total number of SNVs was observed between the alignments of $B$. taurus individuals to each of the reference genomes. Alignment of $B$. taurus individuals against UOA_Brahman_1 resulted in more SNVs than that against ARS UCD1.2 by an average of 5,015,425 more SNVs.

On the contrary, alignment of $B$. indicus individuals against ARS_UCD1.2 resulted in 895,850 more SNVs than that against UOA_Brahman_1, except for the Bohai breed, for which $\sim 3$ million more SNVs were found in the alignment against UOA_Brahman_1 than in that against ARS_UCD1.2. Initially, there was no significant difference ( $p$-value $=0.186$ ) in the total number of SNVs in the alignment of B. indicus individuals

Table 2 Single nucleotide variants (SNVs) for each breed

\begin{tabular}{|c|c|c|c|}
\hline Breeds & Sub-species ${ }^{a}$ & ARS_UCD $1.2^{\mathrm{b}}$ & UOA_Brahman_1 ${ }^{\mathrm{b}}$ \\
\hline Angus & Bos taurus taurus & $11,396,537$ & $17,143,672$ \\
\hline Hereford & Bos taurus taurus & $9,971,428$ & $16,734,834$ \\
\hline Holstein & Bos taurus taurus & $11,133,911$ & $11,459,171$ \\
\hline Jersey & Bos taurus taurus & $9,512,074$ & $15,423,670$ \\
\hline Shorthorn & Bos taurus taurus & $8,464,487$ & $14,393,682$ \\
\hline Simmental & Bos taurus taurus & $14,988,534$ & $20,404,489$ \\
\hline Bohaic $^{c}$ & Bos taurus indicus & $16,102,113$ & $19,819,636$ \\
\hline Boran & Bos taurus indicus & $27,861,344$ & $27,757,944$ \\
\hline Brahman & Bos taurus indicus & $33,177,201$ & $30,592,708$ \\
\hline Gir & Bos taurus indicus & $24,288,498$ & $22,426,843$ \\
\hline Indianzebu & Bos taurus indicus & $20,210,906$ & $18,518,614$ \\
\hline Kenana & Bos taurus indicus & $26,760,622$ & $24,953,316$ \\
\hline Mangshi & Bos taurus indicus & $21,993,501$ & $20,859,379$ \\
\hline Nelore & Bos taurus indicus & $22,770,177$ & $21,279,805$ \\
\hline Ogaden & Bos taurus indicus & $25,330,919$ & $24,224,387$ \\
\hline
\end{tabular}

${ }^{\text {a }}$ Sub-species were assigned based on original labels from NCBI-SRA

${ }^{b}$ SNVs passing the variants filtration process respective to the used reference genome

${ }^{\mathrm{C}}$ Highly admixed with $B$. taurus ancestry 
against either reference genome. However, when we reassigned Bohai as taurine, as the data below suggested was appropriate, we found a significant difference in the number of SNVs (p-value $=0.00069$ ) for B. indicus individuals with an average of 1,472,522 more SNVs called when they were aligned against ARS_UCD1.2 than against UOA_Brahman_1. For B. taurus individuals, this significance increased ( $\mathrm{p}$-value $=0.0011$ ) with an average of 4,830,010 fewer SNVs called when they were aligned against ARS_UCD1.2 than against UOA_Brahman_1.

In the next section, we assessed, separately for each reference genome, how it affected the numbers of NFAA sites instead of the numbers of SNVs in the respective $B$. taurus and $B$. indicus breeds.

\section{Number of NFAA sites obtained in the alignment against ARS_UCD1.2}

The distribution of the number of NFAA sites obtained from the alignments of individuals to the ARS_UCD1.2 assembly is shown in Fig. 2. As expected, the alignment of $B$. indicus sequences against this assembly produced more NFAA sites than that of $B$. taurus sequences, which is consistent with the trend observed in the detection of SNVs. Switching the three Bohai individuals from $B$.

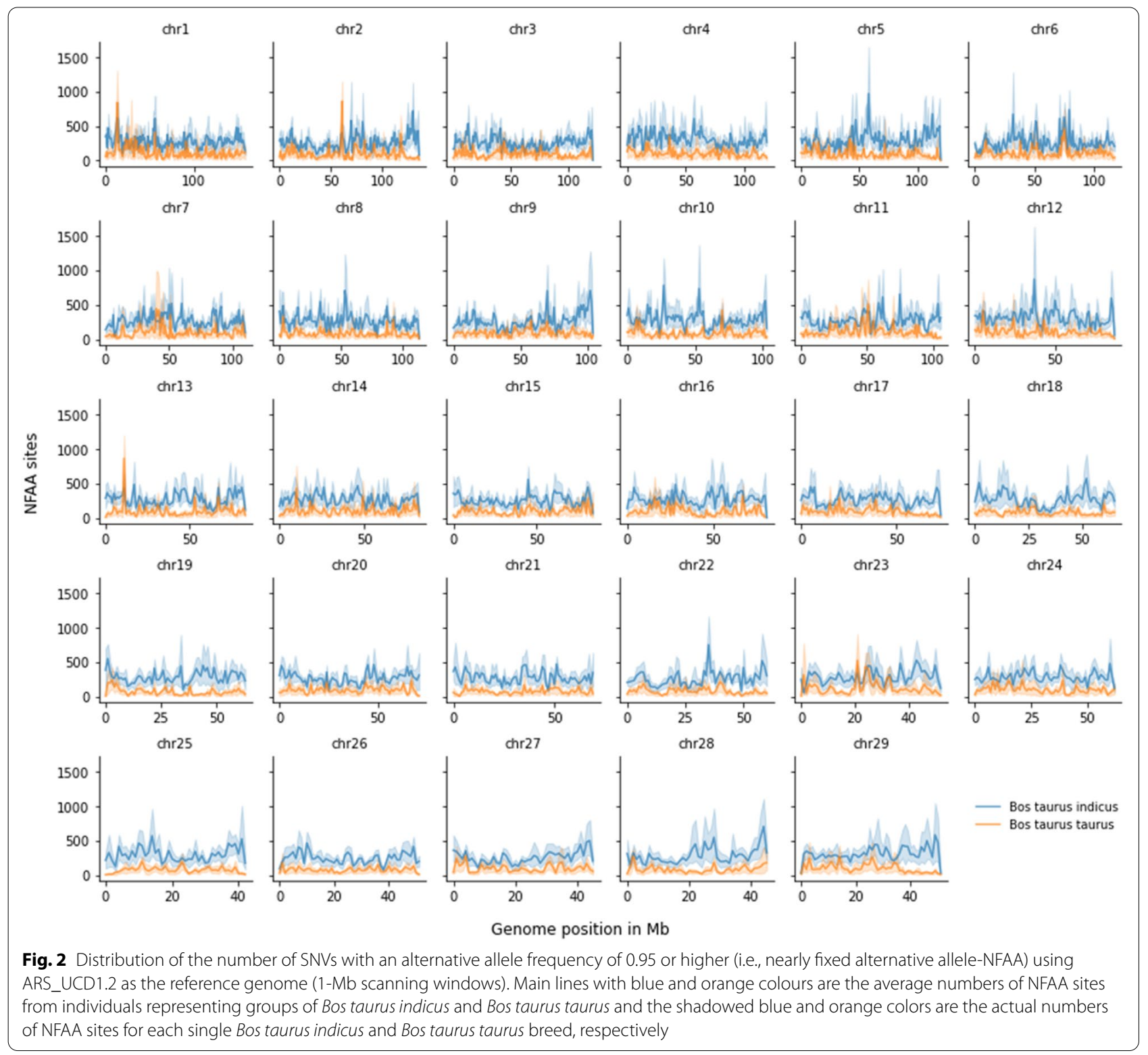


indicus to B. taurus did not change this distribution (see Additional file 2: Fig. S2).

An average number of 242 NFAA sites was found in the indicine data across all analysed windows, with a standard deviation of 96 per $\mathrm{Mb}$. The largest number of NFAA sites (901) was on chromosome 5, around the position at $58 \mathrm{Mb}$, representing approximately one NFAA site per kb. The exceptionally large number of variants in this region extended to the neighbouring chromosomal windows, which presented 366 and 496 NFAA sites. Conversely, we found an average of only $95 \pm 68$ NFAA sites per Mb in the taurine data. The largest number of NFAA sites was 863 , which were located around the position at $11 \mathrm{Mb}$ on chromosome 13. We provide the Z-score transformation of the NFAA sites for alignments to ARS_UCD1.2 in Additional file 2: Fig. S3.

We found a larger number of NFAA sites for indicine than for taurine cattle, regardless of chromosomal position, when reads were aligned to ARS_UCD1.2 (see Additional file 2: Fig. S4). The B. indicus group had three times as many NFAA sites than $B$. taurus across all the autosomes: $\sim 690,000$ sites in $B$. indicus and $\sim 238,000$ sites in B. taurus.

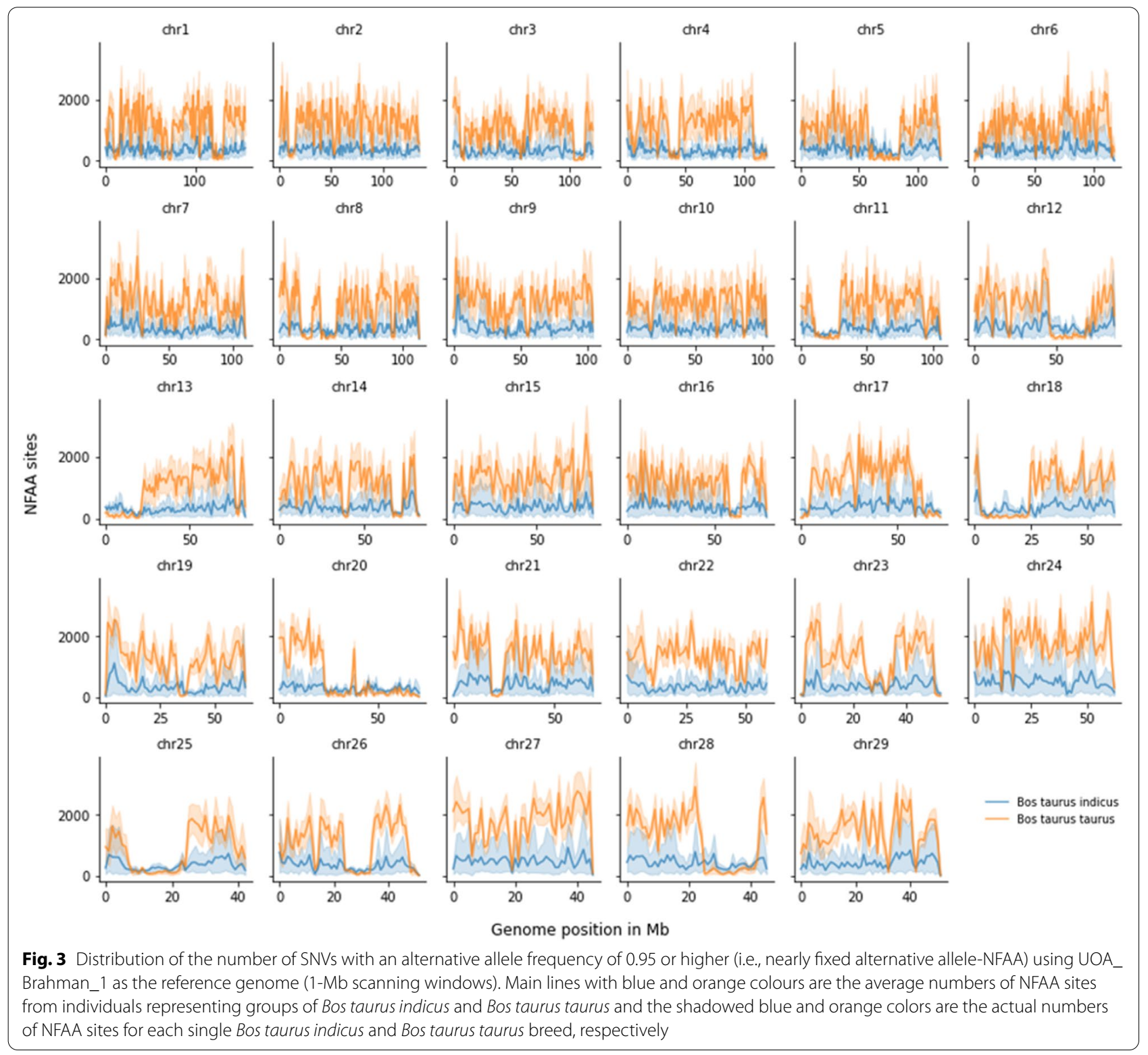




\section{Number of NFAA sites obtained in the alignment against UOA_Brahman_1}

The distribution of the number of NFAA sites based on the UOA_Brahman_1 assembly is shown in Fig. 3. Similar to the results in the previous section, for the more distantly related taurine individuals, the number of NFAA sites (average $1162 \pm 633$ per $\mathrm{Mb}$ ) was larger than for the indicine individuals (average $345 \pm 163$ per $\mathrm{Mb}$ ). Again, classifying the three Bohai individuals as $B$. taurus instead of $B$. indicus did not change this distribution (see Additional file 2: Fig. S5). For B. taurus, the window at $52 \mathrm{Mb}$ on chromosome 24 had the largest number of NFAA sites, with a count of 3105 ( 3 NFAA sites per kb). Additional file 2: Fig. S6 provides a comparison of the boxplot distributions of NFAA sites between $B$. taurus and $B$. indicus.

In addition to the expected results above, we found unique patterns in the alignment against UOA_Brahman_1, which we did not observe in the alignment against ARS_UCD1.2 and which included windows with a small number of NFAA sites for the taurine individuals. Based on our premise that the alignment of less divergent sequences produces smaller numbers of NFAA sites, we conclude that these regions of reduced NFAA counts for B. taurus individuals in the alignment against UOA_Brahman_1 identify traces of taurine-introgression in this assembly. These patterns were confirmed in the Z-score transformation of the raw values of NFAA sites (see Additional file 2: Fig. S7).

\section{B. taurus introgression in the UOA_Brahman_1 assembly}

We extracted the suspected taurine-introgressed regions in the UOA_Brahman_1 assembly by applying Notation 2 and Notation 3 consecutively, as described in the Methods section. Briefly, the $\Delta$ value is a measure of the difference in the number of NFAA sites in a window and the average number across all autosomes. Values of $\Delta$ are visualized in Fig. 4. Regions with $\Delta$ values greater than 1.5 sd from the mean were considered taurine introgressed regions. These putatively taurine-introgressed regions were found on all the autosomes except chromosome 22 and represented 343 of the 2493 one-Mb windows, which corresponds to $13.7 \%$ of the whole genome. By compacting continuous putatively taurine-introgressed regions, this number decreased to 100 segments (see Additional file 3). Forty-seven of these 100 segments were short, i.e. one $\mathrm{Mb}$, and the two longest segments spanned $22 \mathrm{Mb}$ on chromosomes 12 (between positions 47 and $69 \mathrm{Mb}$ ) and 18 (between positions 3 and $25 \mathrm{Mb}$ ).

\section{PCA and admixture analyses}

We used PCA and admixture to validate the position of the Brahman haplotype relative to the genotypes of the other individuals. Figure $5 \mathrm{a}-\mathrm{c}$ shows the clustering of B. taurus and B. indicus individuals using SNVs that were derived from the whole genome, the putativeintrogressed regions, and the non-introgressed regions in UOA_Brahman_1. The variance explained by principal components 1 and 2 were $72 \%$ and $9 \%, 30 \%$ and $8 \%$, and $75 \%$ and $10 \%$ for each respective PCA. Blue arrows on Fig. 5 show the position of the Brahman haplotype relative to that of the other individuals. In Fig. 5a and c, which is based on SNVs from the whole genome and non-introgressed regions, the Brahman haplotype is positioned in the lower right of the graph, closest to the $B$. indicus individuals. Conversely, in Fig. 5b, which is based only on SNVs from genome regions flagged as taurine-introgressed, the Brahman haplotype is positioned in the upper left of the graph, closest to the $B$. taurus cluster. It should be noted that the Bohai breed clusters with the $B$. taurus individuals in all three plots.

The results of the admixture analysis using the SNVs from the whole genome $(\sim 11$ million SNVs), the putative taurine-introgressed $(\sim 801,000 \mathrm{SNVs})$, and the non-introgressed regions $(\sim 10$ million SNVs) in the UOA_Brahman_1 assembly are in Fig. 6a-c, respectively. Figure $6 \mathrm{a}$ and $\mathrm{c}$ show that the Brahman haplotype is again positioned closest to the $B$. indicus individuals, with an admixture pattern that is very similar to that of the B. indicus Boran, Brahman, Gir, Indian zebu, Kenana, Mangshi, Nelore and Ogaden breeds, while Fig. 6b shows that it has a population structure similar to that of the B. taurus Angus, Hereford, Holstein, Jersey, Shorthorn and Simmental breeds. In addition, these admixture analyses suggests that there are still fractions of the $B$. indicus genome, up to a tenth, in the flagged introgressed-regions of the UOA_Brahman_1 reference genome. Figure 6d, which is derived from SNVs $(\sim 17$ million SNVs) obtained in the alignment against ARS_UCD1.2, shows similar patterns of $\mathrm{K}$ distributions among the breeds as that inferred from the taurine-introgressed regions in Fig. 6b. Again, the Bohai breed appears to be more similar to B. taurus than $B$. indicus. In the Admixture software, we chose $K=3$ for the plots in Fig. 6 because it resulted in the lowest cross-validation errors, i.e. 0.632, 0.746, 0.621, and 0.652 for all the SNVs from the whole genome, from the putative taurine-introgressed and the nonintrogressed regions obtained in the alignment against UOA_Brahman_1, and from the whole genome obtained in the alignment against ARS_UCD1.2, respectively (see Additional file 2: Fig. S8). 


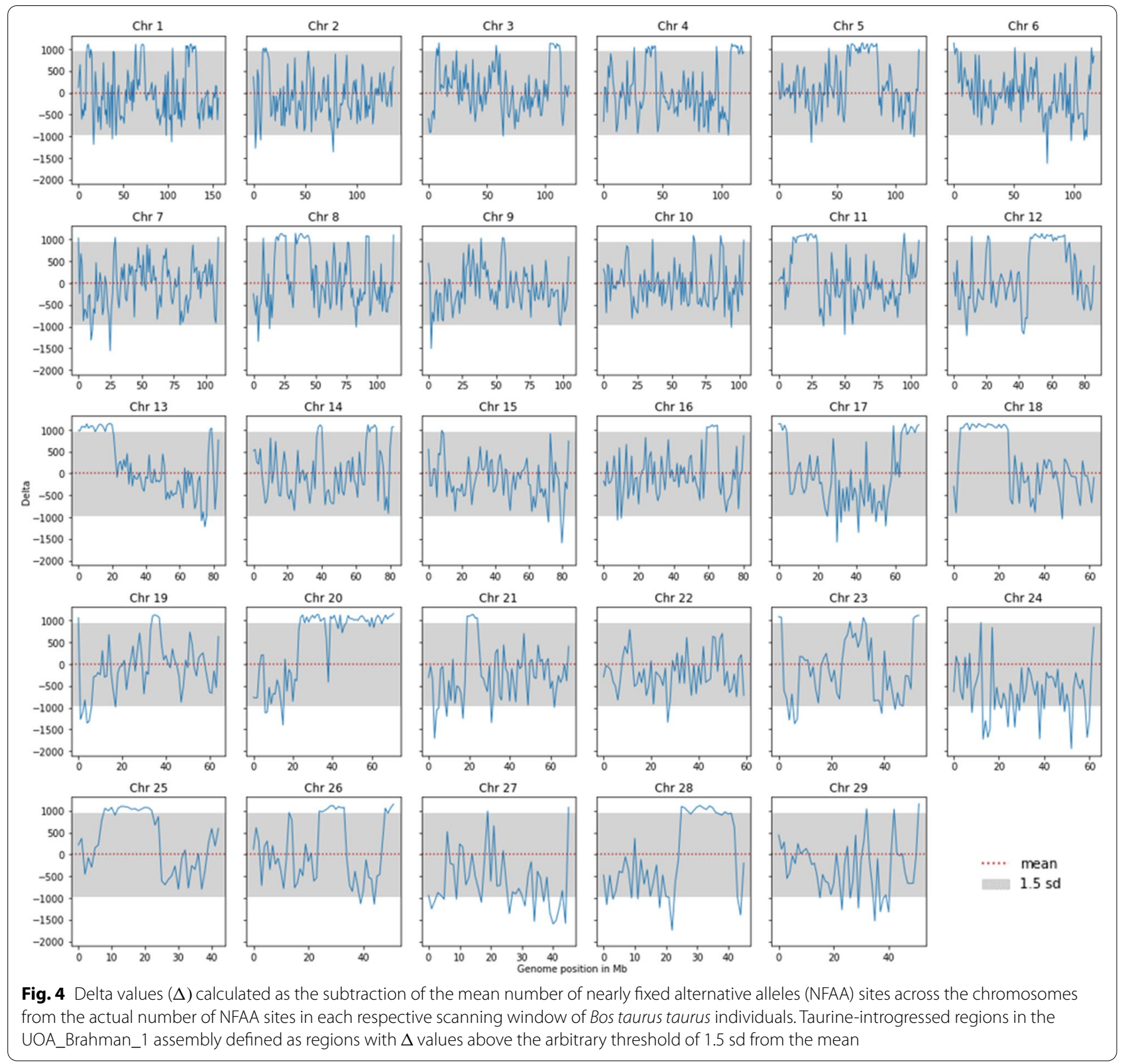

\section{Annotation of taurine-introgressed regions}

We annotated the putative taurine-introgressed regions in the UOA_Brahman_1 assembly and found 2226 genes. The genes for each taurine segment are listed in Additional file 3. Sixty-six of the genes in 19 segments were previously reported to be under positive selection in 40 influential Brahman bulls [11] as shown in Table 3 .

\section{Discussion}

The reference genomes, ARS_UCD1.2 and UOA_ Brahman_1, were built from Hereford-B. taurus and Brahman-B. indicus haplotypes, respectively. Using high-density single nucleotide polymorphisms (SNPs), O'Brien et al. [31] reported a fixation index $\left(\mathrm{F}_{\mathrm{st}}\right.$, i.e. a measure of population differentiation) of 0.374 for Hereford and Brahman populations, which is higher than the within-subspecies $F_{\text {st }}$ values of $0.068,0.160,0.047,0.044$ for Holstein-Hereford, Angus-Hereford, Gir-Brahman, Nelore-Brahman, respectively [31]. Previous studies $[7,18]$ reported that mapping individuals to a reference genome that is built using the same breed resulted in the detection of fewer SNVs than mapping them to a reference genome built from another breed, which is consistent with our results. Indeed, the alignment of B. taurus 


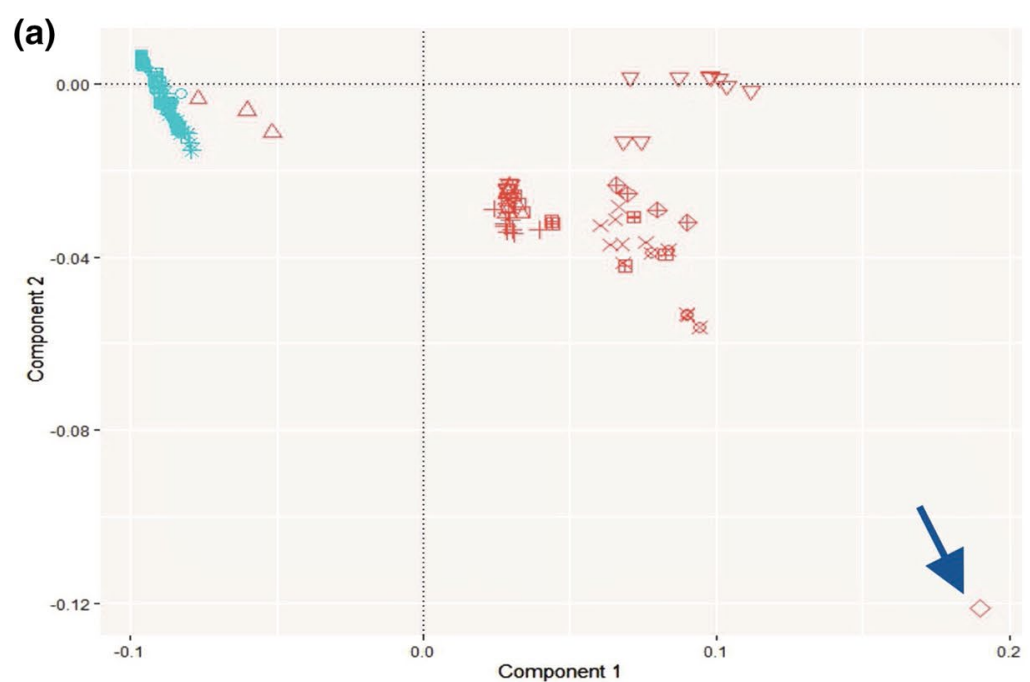

(b)

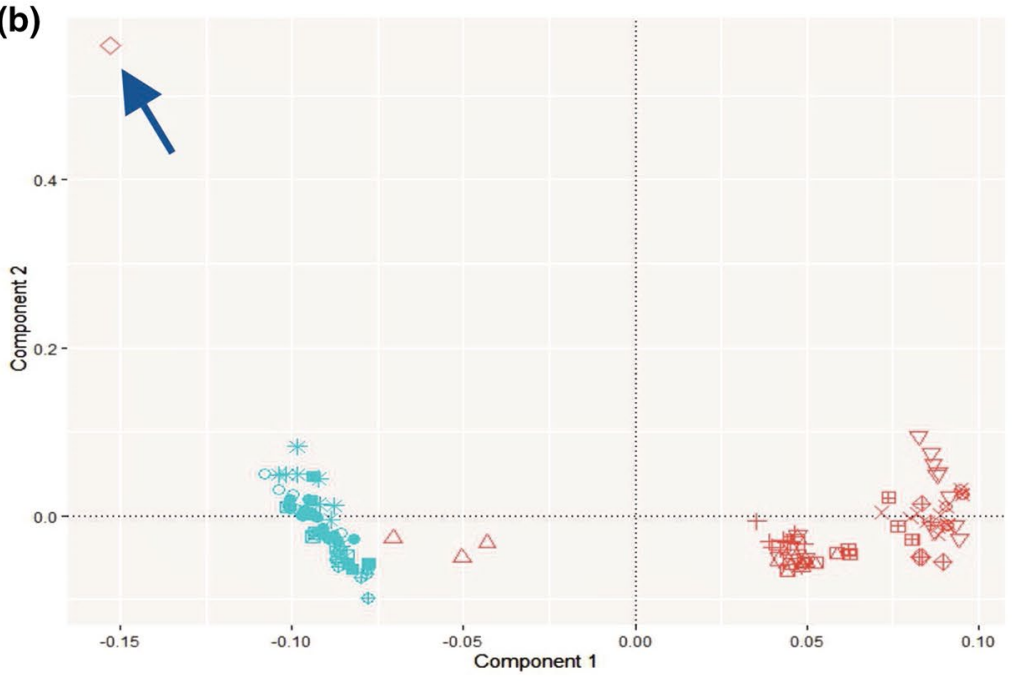

$O$ Angus

$\triangle$ Bohai

+ Boran

$\times$ Brahman

$\diamond$ Brahman_hap

$\nabla$ Gir

$\otimes$ Hereford

* Holstein

$\oplus$ Indian zebu

$\oplus$ Jersey

Kenana

$\boxplus$ Mangshi

Nelore

$\Delta$ Ogaden

- Shorthorn

- Simmental

(c)

- Indicine

- Taurine

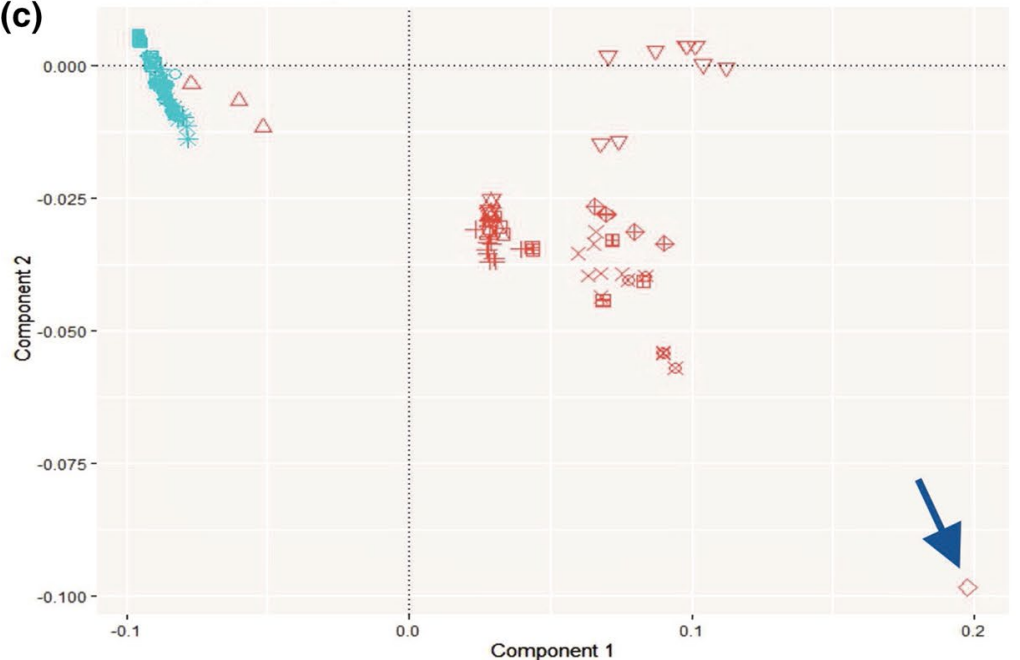

Fig. 5 PCA using SNVs from a the whole genome; b putative taurine-introgressed regions; c non-introgressed regions in the UOA_Brahman_1 assembly. Taurine/indicine were assigned based on original labels from NCBI-SRA. Blue arrows point to the Brahman haplotype (SNVs were set to be all homozygous to the reference alleles of the UOA_Brahman_1 assembly) 

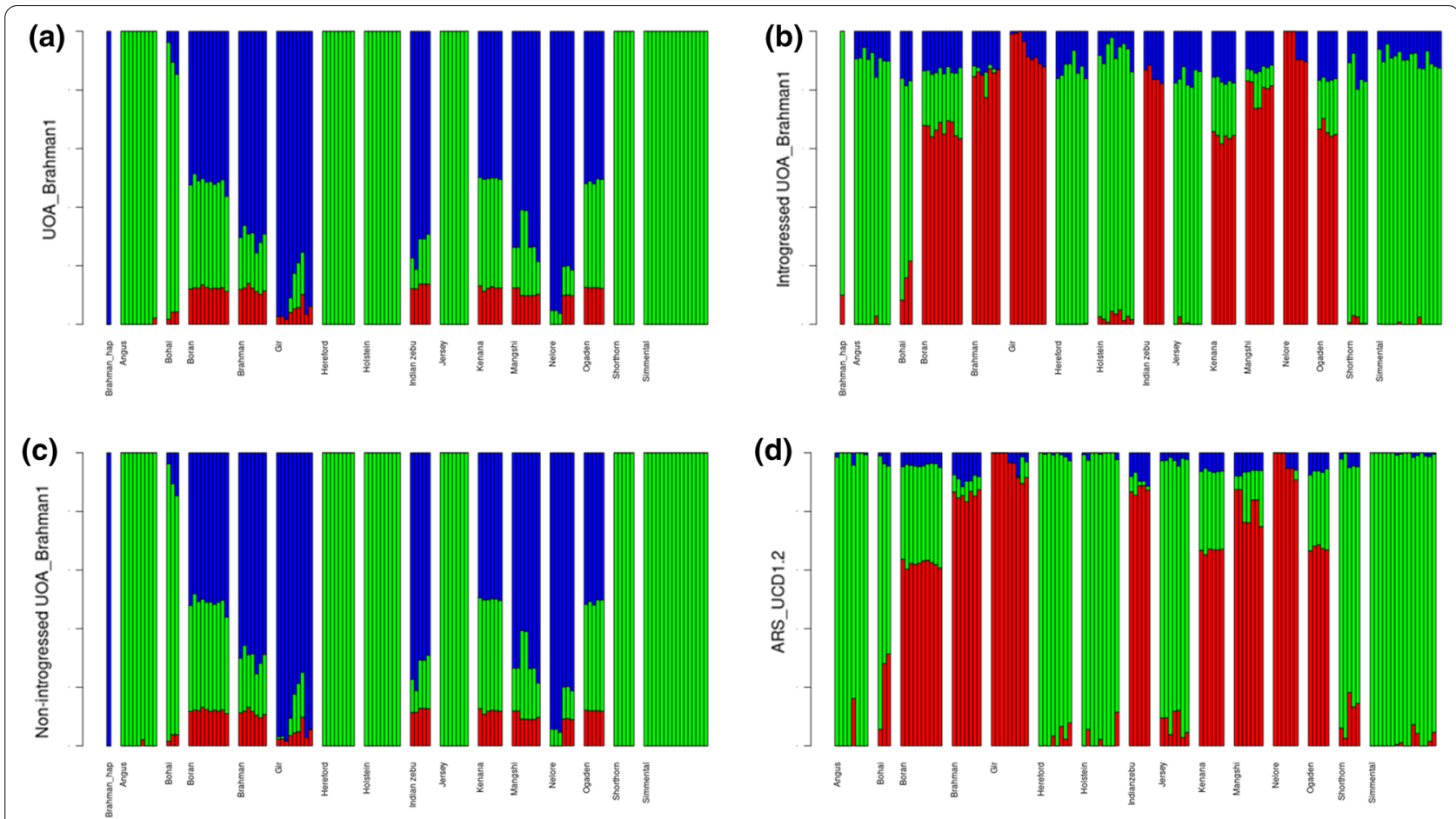

Fig. 6 Admixture analysis using SNVs from $\mathbf{a}$ the whole genome; $\mathbf{b}$ putative taurine-introgressed; $\mathbf{c}$ non-introgressed regions in the UOA Brahman_1 assembly. Brahman_hap is an artificial individual with all the genotypes homozygous to the reference alleles of the UOA_Brahman_1 reference genome. $\mathbf{d}$ Admixture inferred using SNVs derived from the alignment against the ARS_UCD1.2 assembly. Green, red and blue colors depict subpopulations $\mathrm{K} 1, \mathrm{~K} 2$, and $\mathrm{K} 3$, respectively

Table 3 B. taurus introgressed segments in the UOA_Brahman_1 reference genome that overlap with positively selected genes in the Brahman population as reported by [11]

\begin{tabular}{|c|c|c|c|c|c|}
\hline $\mathrm{Chr}$ & Start (Mb) & End (Mb) & Total $\Delta^{\mathrm{a}}$ & Size (Mb) & Positive genes \\
\hline 3 & 58 & 59 & 1090.88 & 1 & BCL10, C1orf52 \\
\hline 5 & 43 & 44 & 1020.05 & 1 & SYT10 \\
\hline 6 & 2 & 4 & 2016.60 & 2 & ANXA5, BBS7, EXOSC9 \\
\hline 7 & 0 & 1 & 1034.72 & 1 & STARD4 \\
\hline 7 & 29 & 30 & 1052.05 & 1 & ACOT12, ANKRD34B, CKMT2, FAM151B, RASGRF2, SSBP2 \\
\hline 10 & 86 & 87 & 1094.05 & 1 & CEP128 \\
\hline 11 & 11 & 13 & 2084.10 & 2 & CYP26B1, DYSF, EMX1, EXOC6B, SPR \\
\hline 11 & 14 & 30 & 17237.41 & 16 & BIRC6, CRIM1, DPY30, MEMO1, NLRC4, SLC3OA6, SPAST, TTC27, YIPF4 \\
\hline 11 & 95 & 96 & 1144.55 & 1 & MAPKAP1, PBX3 \\
\hline 12 & 11 & 12 & 1076.72 & 1 & NAA16, VWA8 \\
\hline 13 & 0 & 21 & 22459.58 & 21 & AHCY, DYNLRB1, MAFB, MAP1LC3A, PIGU, TOP1 \\
\hline 14 & 67 & 68 & 1117.55 & 1 & SDC2 \\
\hline 14 & 81 & 83 & 2138.93 & 2 & SNTB1 \\
\hline 16 & 59 & 66 & 7608.05 & 7 & ZNF648 \\
\hline 17 & 0 & 5 & 5367.15 & 5 & TTC28 \\
\hline 23 & 32 & 33 & 1068.35 & 1 & ABT1, HFE, PRSS16, SLC17A2, TRIM38, ZNF184, ZNF391 \\
\hline 25 & 12 & 23 & 11676.41 & 11 & AQP8, LCMT1, SCNN1G, USP31 \\
\hline 26 & 13 & 14 & 964.55 & 1 & RAB11FIP2 \\
\hline 26 & 24 & 34 & 10574.31 & 10 & $\begin{array}{l}\text { ABCC2, CHUK, COX15, CPN1, CRTAC1, CUTC, DNMBP, ENTPD7, } \\
\text { ERLIN1, SFRP5, SORCS1, ZFYVE27 }\end{array}$ \\
\hline
\end{tabular}


individuals against UOA_Brahman_1 resulted in $\sim 5$ million more SNVs while the alignment of $B$. indicus individuals against ARS_UCD1.2 resulted in only 1.5 million more SNVs. This discrepancy in the number of SNVs detected for cross-subspecies alignments is possibly due to the degree of breed purity. As the results of the admixture analysis show, the $B$. taurus individuals were more uniform while the $B$. indicus individuals displayed a greater level of diversity. It should be noted that the Bohai breed seems to be highly admixed with $B$. taurus ancestry and perhaps should not be labeled as $B$. indicus in the SRA database. The results of the PCA and admixture analysis and the trend of the SNVs detected for this breed followed a pattern similar to those of the B. taurus breeds. In addition, while the alignment of most of the taurine breeds against UOA_Brahman_1 resulted in additional millions of SNVs, that of the Holstein breed resulted in only 300,000 more SNVs. This number of additional SNVs obtained in the cross-alignment of Holstein against UOA_Brahman_1 is comparable to that obtained when indicine sequences are aligned against ARS_UCD1.2. Nevertheless, our findings support that cross-subspecies alignment leads to the detection of more SNVs in agreement with previous studies $[7,18]$.

As the detected SNVs may contain the reference allele, either in the homozygous or heterozygous state, we further explored the effect of cross-subspecies alignments on the number of SNVs with a frequency of alternative alleles higher than 0.95 (NFAA). Based on the results of SNV detection in cross-subspecies alignments, we expect a similar trend for the NFAA sites. To quantify this, we counted the number of NFAA sites for each breed using non-overlapping $1-\mathrm{Mb}$ windows to reflect the one centimorgan (cM) genetic distance [32]. In the alignments against ARS_UCD1.2, we obtained different numbers of NFAA sites within 1-Mb windows between the B. indicus and $B$. taurus cattle groups. The indicine group presented nearly threefold more NFAA sites than the taurine group. This could be explained by the fact that ARS_UCD1.2 derives from an inbred Hereford cow that belongs to the B. taurus subspecies of cattle. Similarly, in the alignments against UOA_Brahman_1, generally the taurine cattle presented about 3.14-fold more NFAA sites than the indicine cattle. Thus, in addition to an increased number of detected SNVs $[7,18]$, we confirm that the numbers of NFAA sites also increase in cross-subspecies alignments of individuals.

Interestingly, we detected regions with fewer than expected NFAA sites by mapping $B$. taurus individuals against UOA_Brahman_1, and suggest that these regions are in fact the result of taurine introgression corresponding to approximately $\sim 13.7 \%$ of the genome. These introgressed regions represent a slightly higher percentage than the reported average of $10 \%$ in the Brahman population [7]. Yet, this proportion is still within the range of previously reported estimates among which the maximum taurine proportion found in a single animal was $37 \%$ [7]. Studies on Brahman populations reported that $B$. taurus ancestry is present on almost all the chromosomes, with a greater proportion of the segments found on chromosomes 1, 4, 8, and 14 [12] and chromosomes 8, 12, 14, 23, 26 and 29 [11]. Consistent with these results, in the alignment against the UOA_Brahman_1 assembly, we found taurine-introgressed regions on all the chromosomes except 22. It is important to note that our proposed taurine-introgressed regions for this assembly correspond to only one haplotype inherited from the maternal lineage of a single animal. Considering the apparent taurine admixture in the Bohai breed, we re-ran the NFAA analysis by assigning Bohai as taurine. However, since only three Bohai individuals were included in our analysis, this did not alter the definition of the taurine introgressed regions in the UOA_Brahman_1 assembly.

Previous studies have aligned WGS data from individuals of admixed and putative-ancestral breeds against a reference sequence and defined regions of introgression as those containing shared derived alleles [11,33]. Similarly, in our study, we used alternative alleles as an indicator of introgression. However, the goals of those previous studies were to identify introgressed regions in individuals relative to the same reference genome, while in our study our aim was to detect introgressed regions in the assembly from the perspective of individuals of known pure-breeds. With the ongoing efforts to construct a consensus genome from multiple individuals to represent the broadest possible diversity within a species $[1,6]$, a similar method of NFAA assessment could be applied to validate that the final consensus genome is not biased.

As supported by the PCA and admixture analyses, the UOA_Brahman_1 haplotype is most similar to that of $B$. taurus individuals in the introgressed regions. The distance of the Brahman haplotype from other samples in the PCA is likely due to sequencing errors in the assembly. The admixture analysis also showed a small fraction of $B$. indicus in the flagged taurine-introgressed regions, which sums up to $10 \%$ of the size of the taurine-introgressed regions and could be explained in two ways: (1) the threshold, which was set at the mean plus 1.5 standard deviation of the $\Delta$ values and used to define the taurine-introgressed regions, may be too lax; and (2) our $1-\mathrm{Mb}$ window size could be too large. Thus, this fraction may, in fact, represent false positives in our analysis.

The longest spans of putative taurine-introgressed regions in this assembly were $22 \mathrm{Mb}$ long on chromosomes 12 and 18 . Considering all the segments, the average length of introgressed regions is $3.43 \mathrm{Mb}$. The length 
of genomic regions with inter-species introgression is strongly related to the history of the breeds since their initial crossing, the breeding scheme, the recombination rates, and the pressure of adaptive/positive selection [11, $12,34,35]$.

We found 2226 genes within putative $B$. taurus regions in the UOA_Brahman_1 assembly. As we flagged 13.7\% of UOA_Brahman_1 assembly as taurine-introgressed regions, the number of genes found within these regions was smaller by 823 than the number expected by chance. These 2226 genes were classified into two groups: (1) a group of 66 genes that have been reported to be under positive selection [11], with functions related to productivity and developments traits, as shown in the Results section; and (2) a group of neutral genes that are in randomly segregating putative $B$. taurus regions, since they have not been reported to be under positive selection in Brahman cattle. Although unreported, these genes might be important for the individual itself, i.e. related to productivity and developments traits, which were selected for in the formation of Brahman cattle, such as: SYT10, a gene involved in feed efficiency [36]; BIRC6 the expression of which is considered vital for embryo survival particularly during pre-implantation embryonic development in cattle [37]; CYP26B1, which encodes a retinoic acid metabolizing enzyme; it has been shown that CYP26B1 knock-out impacts the skeletal and limb formation in vertebrate embryos $[38,39]$; genes related to binding activities for phospholipid and ATP as indicated by MAP1LC3A, MAPKAP1, and SPAST; and NLRC4 and HFE related to the major histocompatibility complex (MHC) involved in immune responses.

The number of identified SNVs and their allele frequencies in any sample pool are prone to be altered by the individual's background such as breed type, size, phenotypic performance, relatedness, and also by the software chosen for calling variants [40-44]. Although, we used a relatively small subset of individuals and only a single tool for calling variants (i.e. GATK) to infer the presence of putative $B$. taurus sequences in the UOA_Brahman_1 assembly, we presume that these segments can result in the detection of more SNVs with nearly fixed alternative alleles in the alignments of novel B. indicus individuals. This has the potential to falsely identify signatures of selection because the number of alternative alleles, also called derived alleles, is frequently used to identify signatures of selection [45-47].

Choosing one reference assembly over another can lead to trade-offs in downstream genomic analyses. As indicated by our results, aligning individuals against different reference assemblies results in different numbers of detected SNVs and different allele frequencies which are crucial for the accuracy of genome-wide association studies and of analyses of signatures of selection [4548]. Other points to consider are how well the reference genome builds are supported in various genomic tools and how often is the reference genome used by other studies to enable straightforward comparisons of results [5]. The main reason to switch from ARS_UCD1.2 to UOA_Brahman_1 as the reference genome is to improve sequence read mapping and avoid possible variant calling bias for re-sequencing $B$. indicus individuals. Thus, downstream analysis can be carried out more accurately, since the individual that is used to reconstruct the assembly better represents the breed. Therefore, we highly recommended the use of UOA_Brahman_1 as the reference genome for the analysis of re-sequencing $B$. indicus individuals keeping in mind that it contains taurine segments that need to be accounted for.

\section{Conclusions}

Our results confirm that an increased genetic distance between the sequence data to be aligned and the chosen reference genome results in an increased number of detected variants. We flagged $13.7 \%$ of the genome in UOA_Brahman_1 as originating from Bos taurus taurus. The genes that are located within these taurine introgressed segments and are reported to be under positive selection in the Brahman population are linked to feed efficiency, reproduction, and immune response traits. These reported taurine-introgressed segments should be taken into consideration in forthcoming re-sequencing analyses of indicine genomes aligned to the UOA_Brahman_1 assembly since they might cause variant calling bias.

\section{Supplementary Information}

The online version contains supplementary material available at https://doi. org/10.1186/s12711-021-00688-1.

Additional file 1: Table S1. Accession numbers and alignment details. The table provides the accession numbers of all individuals used as representatives of $B$. taurus and $B$. indicus, as well as details for alignments against both reference genomes (ARS_UCD1.2 and UOA_Brahman_1).

Additional file 2: Figure S1. Density function of $\Delta$ values. Figure S2. Distribution of nearly fixed alternative allele-NFAA sites considering Bohai as taurine for alignment against ARS_UCD1.2. Figure S3. Z-score transformation of nearly fixed alternative allele-NFAA sites distribution for alignment against ARS_UCD1.2. Figure S4. Boxplot of nearly fixed alternative allele-NFAA sites for alignment against ARS_UCD1.2. Figure S5. Distribution of nearly fixed alternative allele-NFAA sites considering Bohai as taurine for alignment against UOA_Brahman_1. Figure S6. Boxplot of nearly fixed alternative allele-NFAA sites for alignment against UOA_Brahman_1. Figure S7. Z-score transformation of the nearly fixed alternative allele-NFAA sites distribution for alignment against UOA_Brahman_1. Figure S8. Cross-validation error for the admixture analysis.

Additional file 3. Putative segments of $B$. taurus introgressed regions in the UOA_Brahman_1 reference genome. The data is in semicolonseparated format. It provides information for the physical location of 100 
segments flagged as B. taurus introgressed regions in the UOA_Brahman_1 assembly and their delta values and annotated genes. Positive genes are those reported in [1 1], and neutral genes are found within each respective segment, but are not listed in [1 1].

\section{Acknowledgements}

Computation was carried out in Vienna Scientific Cluster (VSC3).

\section{Authors' contributions}

GM, YTU, and BDR conceived and designed the study. MMN ran the analysis and drafted the manuscript. YTU, BDR, JS, and GM interpreted the analysis results and critically revised the manuscript. All authors read and approved the final manuscript.

\section{Funding}

MMN is supported by the Ernst Mach Grant, ASEA UNINET (OeAD Austria).

\section{Availability of data and materials}

Accession numbers for each individual used in this study are provided in 'Additional file 1: Table S1'and can be retrieved from the NCBI repository https:// www.ncbi.nlm.nih.gov/sra/: Cattle reference sequences ARS_UCD1.2 (B. taurus) are available at the NCBI repository https://www.ncbi.nlm.nih.gov/genom e/?term=cattle. Cattle reference sequences UOA_Brahman_1 (B. indicus) are available at the NCBI repository https://www.ncbi.nlm.nih.gov/assembly/ GCF_003369695.1. Codes and in-house Python scripts are available at https:// github.com/mas-agis/taurus_segment_uoa_brahman.git.

\section{Declarations}

\section{Ethics approval and consent to participate}

Not applicable.

\section{Consent for publication}

Not applicable.

\section{Competing interests}

The authors declare that they have no competing interests.

\section{Author details}

'University of Natural Resources and Life Sciences (BOKU), Vienna, Austria. ${ }^{2}$ AgroPartners Consulting, R. Floriano Peixoto, 120 - Sala 43A - Centro, Araçatuba, SP 16010-220, Brazil. ${ }^{3}$ Department of Production and Animal Health, School of Veterinary Medicine, São Paulo State University (Unesp), Araçatuba, São Paulo, Brazil. ${ }^{4}$ International Atomic Energy Agency (IAEA) Collaborating Centre on Animal Genomics and Bioinformatics, Araçatuba, São Paulo, Brazil. ${ }^{5}$ Animal Genomics and Improvement Laboratory, USDA, ARS, Beltsville, MD, USA.

Received: 12 March 2021 Accepted: 29 November 2021

Published online: 18 December 2021

\section{References}

1. Ballouz S, Dobin A, Gillis JA. Is it time to change the reference genome? Genome Biol. 2019;20:159.

2. 1000 Genomes Project Consortium, Auton A, Brooks LD, Durbin RM, Garrison EP, Kang HM, et al. A global reference for human genetic variation. Nature. 2015;526:68-74.

3. Altmann A, Weber P, Bader D, Preuss M, Binder EB, Muller-Myhsok B. A beginners guide to SNP calling from high-throughput DNA-sequencing data. Hum Genet. 2012;131:1541-54.

4. DePristo MA, Banks E, Poplin R, Garimella KV, Maguire JR, Hartl C, et al. A framework for variation discovery and genotyping using next-generation DNA sequencing data. Nat Genet. 2011;43:491-8.

5. Van der Auwera GA, Carneiro MO, Hartl C, Poplin R, Del Angel G, Levy-Moonshine A, et al. From FastQ data to high confidence variant calls: the Genome Analysis Toolkit best practices pipeline. Curr Protoc Bioinform. 2013:43:11.

6. Heaton MP, Smith TPL, Bickhart DM, Vander Ley BL, Kuehn LA, Oppenheimer J, et al. A reference genome assembly of Simmental cattle, Bos taurus taurus. J Hered. 2021;112:184-91.

7. Low WY, Tearle R, Liu R, Koren S, Rhie A, Bickhart DM, et al. Haplotyperesolved genomes provide insights into structural variation and gene content in Angus and Brahman cattle. Nat Commun. 2020;11:2071.

8. Crysnanto D, Pausch H. Bovine breed-specific augmented reference graphs facilitate accurate sequence read mapping and unbiased variant discovery. Genome Biol. 2020;21:184.

9. Rosen BD, Bickhart DM, Schnabel RD, Koren S, Elsik CG, Tseng E, et al. De novo assembly of the cattle reference genome with single-molecule sequencing. GigaScience. 2020;9:giaa021.

10. Zimin AV, Delcher AL, Florea L, Kelley DR, Schatz MC, Puiu D, et al. A whole-genome assembly of the domestic cow, Bos taurus. Genome Biol. 2009;10:R42.

11. Koufariotis L, Hayes BJ, Kelly M, Burns BM, Lyons R, Stothard P, et al. Sequencing the mosaic genome of Brahman cattle identifies historic and recent introgression including polled. Sci Rep. 2018:8:17761.

12. Bolormaa S, Hayes BJ, Hawken RJ, Zhang Y, Reverter A, Goddard ME. Detection of chromosome segments of zebu and taurine origin and their effect on beef production and growth. J Anim Sci. 2011;89:2050-60.

13. Hansen PJ. Physiological and cellular adaptations of zebu cattle to thermal stress. Anim Reprod Sci. 2004;82-83:349-60.

14. Achilli A, Olivieri A, Pellecchia M, Uboldi C, Colli L, Al-Zahery N, et al. Mitochondrial genomes of extinct aurochs survive in domestic cattle. Curr Biol. 2008;18:R157-8.

15. Murray C, Huerta-Sanchez E, Casey F, Bradley DG. Cattle demographic history modelled from autosomal sequence variation. Philos Trans R Soc Lond B Biol Sci. 2010:365:2531-9.

16. Fernandes Júnior GA, de Oliveira HN, Carvalheiro R, Cardoso DF, Fonseca LFS, Ventura RV, et al. Whole-genome sequencing provides new insights into genetic mechanisms of tropical adaptation in Nellore (Bos primigenius indicus). Sci Rep. 2020;10:9412.

17. Iqbal N, Liu X, Yang T, Huang Z, Hanif Q, Asif M, et al. Genomic variants identified from whole-genome resequencing of indicine cattle breeds from Pakistan. PLoS One. 2019;14:e0215065.

18. Bovine HapMap Consortium, Gibbs RA, Taylor JF, Van Tassell CP, Barendse W, Eversole KA, et al. Genome-wide survey of SNP variation uncovers the genetic structure of cattle breeds. Science. 2009;324:528-32.

19. Li H, Durbin R. Fast and accurate long-read alignment with BurrowsWheeler transform. Bioinformatics. 2010;26:589-95.

20. Li H, Handsaker B, Wysoker A, Fennell T, Ruan J, Homer N, et al. The sequence alignment/map format and SAMtools. Bioinformatics. 2009;25:2078-9.

21. McKenna A, Hanna M, Banks E, Sivachenko A, Cibulskis K, Kernytsky A, et al. The Genome Analysis Toolkit: a MapReduce framework for analyzing next-generation DNA sequencing data. Genome Res. 2010;20:1297-303.

22. Hayes BJ, Daetwyler HD. 1000 Bull Genomes project to map simple and complex genetic traits in cattle: applications and outcomes. Annu Rev Anim Biosci. 2019;7:89-102.

23. Wu DD, Ding XD, Wang S, Wójcik JM, Zhang Y, Tokarska M, et al. Pervasive introgression facilitated domestication and adaptation in the Bos species complex. Nat Ecol Evol. 2018;2:1139-45.

24. Hoff JL, Decker JE, Schnabel RD, Taylor JF. Candidate lethal haplotypes and causal mutations in Angus cattle. BMC Genomics. 2017;18:799.

25. Danecek P, Auton A, Abecasis G, Albers CA, Banks E, DePristo MA, et al. The variant call format and VCFtools. Bioinformatics. 2011;27:2156-8.

26. Chang CC, Chow CC, Tellier LC, Vattikuti S, Purcell SM, Lee JJ. Secondgeneration PLINK: rising to the challenge of larger and richer datasets. GigaScience. 2015;4:7.

27. Wickham H. ggplot2: Elegant graphics for data analysis. New York: Springer-Verlag; 2016

28. R Core Team. R: A language and environment for statistical computing. Vienna: R Foundation for Statistical Computing; 2020.

29. Alexander DH, Novembre J, Lange K. Fast model-based estimation of ancestry in unrelated individuals. Genome Res. 2009;19:1655-64.

30. Cingolani P, Platts A, Wang LL, Coon M, Nguyen T, Wang L, et al. A program for annotating and predicting the effects of single nucleotide 
polymorphisms, SnpEff: SNPs in the genome of Drosophila melanogaster strain w1118; iso-2; iso-3. Fly (Austin). 2012;6:80-92.

31. O'Brien AMP, Höller D, Boison SA, Milanesi M, Bomba L, Utsunomiya YT, et al. Low levels of taurine introgression in the current Brazilian Nelore and Gir indicine cattle populations. Genet Sel Evol. 2015;47:31.

32. Andersson L, Georges M. Domestic-animal genomics: deciphering the genetics of complex traits. Nat Rev Genet. 2004;5:202-12.

33. Chen N, Cai Y, Chen Q, Li R, Wang K, Huang Y, et al. Whole-genome resequencing reveals world-wide ancestry and adaptive introgression events of domesticated cattle in East Asia. Nat Commun. 2018;9:2337.

34. Medugorac I, Graf A, Grohs C, Rothammer S, Zagdsuren Y, Gladyr E, et al. Whole-genome analysis of introgressive hybridization and characterization of the bovine legacy of Mongolian yaks. Nat Genet. 2017:49:470-5.

35. Barbato M, Hailer F, Upadhyay M, Del Corvo M, Colli L, Negrini R, et al. Adaptive introgression from indicine cattle into white cattle breeds from Central Italy. Sci Rep. 2020;10:1279.

36. Barendse W, Reverter A, Bunch RJ, Harrison BE, Barris W, Thomas MB. A validated whole-genome association study of efficient food conversion in cattle. Genetics. 2007;176:1893-905.

37. Salilew-Wondim D, Hölker M, Rings F, Phatsara C, MohammadiSangcheshmeh A, Tholen E, et al. Depletion of BIRC6 leads to retarded bovine early embryonic development and blastocyst formation in vitro. Reprod Fertil Dev. 2010;22:564-79.

38. Dranse HJ, Sampaio AV, Petkovich M, Underhill TM. Genetic deletion of Cyp26b1 negatively impacts limb skeletogenesis by inhibiting chondrogenesis. J Cell Sci. 2011;124:2723-34.

39. Cunningham TJ, Duester $\mathrm{G}$. Mechanisms of retinoic acid signalling and its roles in organ and limb development. Nat Rev Mol Cell Biol. 2015;16:110-23.

40. Lin S, Zhang H, Hou Y, Liu L, Li W, Jiang J, et al. SNV discovery and functional candidate gene identification for milk composition based on whole genome resequencing of Holstein bulls with extremely high and low breeding values. PLoS One. 2019;14:e220629.

41. Stafuzza NB, Zerlotini A, Lobo FP, Yamagishi MEB, Chud TCS, Caetano $A R$, et al. Single nucleotide variants and InDels identified from wholegenome re-sequencing of Guzerat, Gyr, Girolando and Holstein cattle breeds. PLoS One. 2017;12:e0173954.

42. Naji MM, Utsunomiya YT, Sölkner J, Rosen BD, Mészáros G. Investigation of ancestral alleles in the Bovinae subfamily. BMC Genomics. 2021;22:108.

43. Zwane AA, Schnabel RD, Hoff J, Choudhury A, Makgahlela ML, Maiwashe $A$, et al. Genome-wide SNP discovery in indigenous cattle breeds of South Africa. Front Genet. 2019;10:273.

44. Crysnanto D, Wurmser C, Pausch H. Accurate sequence variant genotyping in cattle using variation-aware genome graphs. Genet Sel Evol. 2019;51:21.

45. Ronen R, Tesler G, Akbari A, Zakov S, Rosenberg N, Bafna V. Predicting carriers of ongoing selective sweeps without knowledge of the favored allele. PLoS Genet. 2015;11:e1005527.

46. Field Y, Boyle EA, Telis N, Gao Z, Gaulton KJ, Golan D, et al. Detection of human adaptation during the past 2000 years. Science. 2016;354:760-4.

47. Xu L, Bickhart DM, Cole JB, Schroeder SG, Song J, Tassell CPV, et al. Genomic signatures reveal new evidences for selection of important traits in domestic cattle. Mol Biol Evol. 2015;32:711-25.

48. Goddard ME, Hayes BJ. Mapping genes for complex traits in domestic animals and their use in breeding programmes. Nat Rev Genet. 2009;10:381-91.

\section{Publisher's Note}

Springer Nature remains neutral with regard to jurisdictional claims in published maps and institutional affiliations.

Ready to submit your research? Choose BMC and benefit from:

- fast, convenient online submission

- thorough peer review by experienced researchers in your field

- rapid publication on acceptance

- support for research data, including large and complex data types

- gold Open Access which fosters wider collaboration and increased citations

- maximum visibility for your research: over $100 \mathrm{M}$ website views per year

At BMC, research is always in progress.

Learn more biomedcentral.com/submissions 